\title{
Detection and analysis of Brain Tumor from MRI by Fully Convolutional Neural Network method
}

\author{
T. Sandhya Rani ${ }^{1}$, Vadivelan Natarajan ${ }^{2}$ \\ ${ }^{1}$ PG Scholar,Department of Computer Science and Engineering, \\ ${ }^{2}$ Professor, Department of Computer Science and Engineering, \\ Teegala Krishna Reddy Engineering College, Meerpet, Hyderabad, Telangana 500097
}

Article History:Received:11 november 2020; Accepted: 27 December 2020; Published online: 05 April 2021 Abstract :Although it seems that the technology used in MRI is technologically advanced, we now use MRI to detect tumors based on human skills and knowledge. Although this method is proven to be accurate in most cases, sometimes the human eye does not work until it detects a slight deviation. In countries like India, there are no oncologists or neurologists. According to a report from 2018, the country is facing a shortage of health workers, with only 2,000 cancer specialists treating 10 million patients in rural areas, where the number of cancer specialists is very low, and its condition worse.

\section{Keywords:}

\section{INTRODUCTION}

Tomography and MRI are used to detect brain tumors. MRI uses a magnetic field instead of X-rays to create a complete body image. The true diagnosis is very important in the diagnosis of cerebral palsy. Accuracy has improved with the help of computer systems. This system helps radiation experts to detect frequent brain tumors. In our experiment, we will discuss new ways to calculate the size and location of tumors using a computer to improve the accuracy of detecting brain tumors. Also, it can help whether the tumor is malignant or not. The size, length, location, or thickness of the tumor is also important when a specialist doctor decides to operate. The paper shows how to calculate the location of the tumor. This is an important factor in measuring.

There are two key factors in the diagnosis of cerebral palsy. The basic approach to the boundaries of the universe is based on the idea of determining the universal image bond of the whole image. In this way, images are categorized according to specific boundary values, which divide the image into two groups, one group with a larger size than the other, another group of smaller size or equal to the boundary value, and another group of morphological image processes.

\section{LITERATURE SURVEY}

Instead of detecting brain tumors, Rohan Kandwal and Ashok Kumar proposed a method for diagnosing and classifying tumor rhinoplasty in an automated system, focusing more on the process in this paper. Besides, its text does not comment on accuracy.

Jasdeip Kaur and Mamta Garg proposed "balanced media filters for image enhancement" that improve the performance of balanced media filters or change media filters to see digital gray waves.

Rohit S. Kabade and Drs. MS Gaikwad proposed the method of "Distribution of brain tumors using the K-mean group with unknown C-mean calculations and calculations located in MRI brain images", which can be compared with the manual division of tumor cells. it also reduces analysis time. One of the biggest problems with tumor fragmentation is detecting chronic tumors.

AnamMustakeem, Ali Javed, and Tehsin Fatima proposed the method in the "Alive Alive for Discovering Water Supply and Coastal Distribution", where the authors provide routes based on coastal distribution, run-offs, and genetic operators. His method is recommended for the diagnosis of cerebral palsy, but there is no information about the level of success.

In his paper "Determination and Distribution of Brain Tumors Using Basin Distribution and Morphological Surgery", Swe Zin Oo and Aung SoeKhaing suggest methods for detecting tumors and the morphological effects of erosion algorithms. The main disadvantage of this method is very low accuracy with only $52.78 \%$ efficiency.

Md. Sujan, Sayyid Abdullah Noman, NashidAlam, and M. Jahir al-Islam proposed this method in an "automated system based on the detection of brain tumors." In this way, the image is converted to a gray image, and noise is generated by several filtration parameters. The experimental results show an accuracy of $84.72 \%$ in judging 61 successful images from 72 FLAIR images of the BRATS set. Although the accuracy of this method is high, there is no information about the location or size of its tumor.

Corresponding Author : T. Sandhya Rani,

PG Scholar,Department of Computer Science and Engineering,

Teegala Krishna Reddy Engineering College, Meerpet, Hyderabad, Telangana 500097 
Image sharing methods and techniques work in many industries. The distribution of cerebral palsy and general brain structure is the most important use in medical imaging. Magnetic resonance imaging (MRI) is one of the imaging techniques that can examine and capture the soft tissue structure of the body. The paper shows how to separate the parts of the tumor, successfully propagate the tumor, find the tumor in a circle, and determine if its tumor exists. To facilitate the study and analysis of complex brain structures, a method based on vague groups has been recommended, to be used as a tool for optical analysis and research.

Processing medical images are one of the most difficult research topics. The main purpose of image sharing is to identify different image functions that can be used to analyze, interpret, and understand images. Medical MRI imagination plays an important role in medical diagnosis. Brain imaging is very important to know what type of brain condition is serious, which can reduce the risk of death. This paper provides a good way to share images that can distinguish biological tissues such as tumors and tumors from normal tissues such as white matter (WM), gray (GM), and cerebrospinal fluid. Development is the simplest and most widely used way to share images. This method can judge the swelling of the brain

The photo section divides the image into areas or objects. To some degree, debt transfer depends on the solution of the problem. The distribution of insignificant images is a major problem in image processing. Division accuracy is crucial to the success or failure of automated analytical procedures. The dividing mechanism focuses on the area, not its size. The result of that part is the division of the image into an interconnected area. Image sharing is an important step in analyzing, understanding, interpreting, and identifying images. Distribution is one of the most important steps in automated diagnostic systems, which are widely used in medical imaging, satellite imagery, motion detection, security, monitoring, and much more.

\section{OVERVIEW OF THE SYSTEM}

\subsection{EXISTING SYSTEM:}

Distribution and calculation of brain tumor location in MRI brain images using K-mean and C-mean analgesia algorithms. This method allows tumor tissue to be distributed more accurately and evenly compared to manual division and also reduces analysis time.

An effective algorithm for detecting brain tumors using fluid and boundary divisions, "in which the authors propose methods based on port divisions, river basins, and genetic carriers.".

\section{Disadvantages:}

One of the main problems of tumor division is hand detection, which takes time.

\subsection{PROPOSED SYSTEM:}

The data we use is a pair of BRATS. This database contains images of brain tissue fragmentation. After imaging, there are 3 classes: necrotic and non-invasive tumors (NCR / NET), peritumor tumors (ED), and GD (ET) tumors. The training data set contains about 220 images.

To divide the MRI brain, we use a popular torpedo called U-Net architecture, which is built on a completely convex path and facilitates the smooth separation of medical images. According to FCN-8, the two main differences .U-net is symmetry. The connection between the download and the start line is used instead of the assembly of the operators involved. This adoption link is intended to provide general and worldly news during the experiment. Because of its symmetry, torpedoes have multiple object maps in the experimental path, which facilitates the transfer of information. In comparison, the basic architecture of FCN consists of only a few classrooms made up of road maps.

\section{Advantages:}

To solve this problem, we recommend Brainy, an MRI scanner in the brain. Brain tumors do not alter cognitive processes but help to resolve cases that are not visible to the naked eye.

\section{Architecture:}

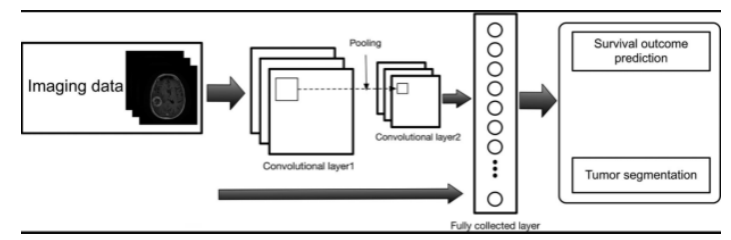

\section{METHODOLOGY:}

Data were collected from the BRATS brain tumor database [8]. There are four types of images for each patient: T1, T2, T1C, and FLAIR. In our experiment, we used FLAIR images of 76 patients. Its database contains 3D images in .mha file format. Use 3D Views to convert 3D images to 2D images in .png format.

\section{A. Histogram Method}

This way we create a gray background image. This gray image uses the technique of launching a histogram to improve the contrast of the image and judge the clear parts of the image. After careful screening, we found images that were smooth and accurate. Before waiting for the Otsu setting of 0.31 , we changed the 
nearby white pixels to full white and some to darker. Then we convert that image into a binary image. We performed morphological expansion and degradation with mixed elements $\left[\begin{array}{lll}1 ; & 1 & 1\end{array}\right]$ this produces better distribution. We release pixels smaller than 250 pixels because it helps to identify areas that are swollen. Finally, tumor location is defined.

Using this process, we allow the following: - Using a histogram, image contrast, in general, is significantly improved. For this purpose, there are white pixels in a light-lit area. - By adding more pixels, we get unnecessary white pixels that are incorrect white pixels. If we divide the affected area based on a large white area, it will be cured. - When we filter, we find missing pixels that are lost due to morphological function. Therefore, to prevent the loss of these tumor pores during morphological processes, we try to restore these pixels. This problem confuses us when we identify areas that are affected. Based on the results of this method, we find that it is inaccurate.

B. Revised Integrated Thresholding and Morphological Method

In this way, the FLAIR image is converted to a gray image. Gray images are stored on exchanges other than Otsu. We use a world-class approach because it allows us to affect areas of the brain as much as possible. First, a universal swimming pool was established. T-shaped images are divided into two groups so that 1dimensional groups are larger than $\mathrm{T}$ and 2-dimensional groups are less than or equal to $\mathrm{T}$. Average weight is calculated from two groups. Finally, the value of the new pool is calculated from the meaning of two extreme values

C. Number Of Tumors

We put the tumor pixels together to form a very white area. With four pathways close to the work of Bwlabel (), we can determine the number of tumors in the brain. Although patients have at least two tumors.

D. Location and Affected Ratio of the Tumor

In the same way, we create white dots from tumors and all brain and calculate the area and function of its nnz ().

We split the image into four smaller images. Je! Are there four boxes on the market? Then we calculate the pixels for each child's image. Among the registrars, we define tumor locations with a large number of boundaries. Then we can say that the location of the tumor depends on the maximum area between these numbers.

E. Malignancy

If there are two tumors in the brain, we can assume that the tumor is very malignant. But it is difficult to distinguish crime from images alone. Because there is a reason why patients are sick. For example, age, clinical picture, if the patient has a swollen childhood, current tumor status, and so on.

\section{OUTPUT SCREEN SHOTS}

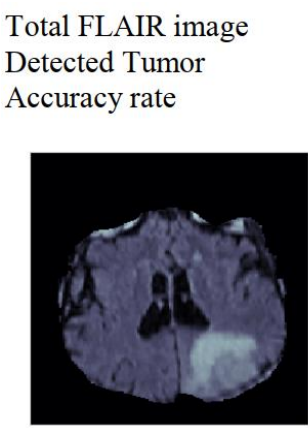

(a)
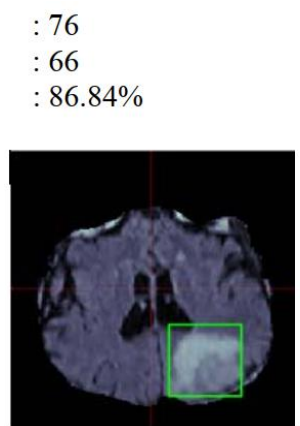

(b)

Fig 9: (a) Input FLAIR Image, (b) Generated Output Image

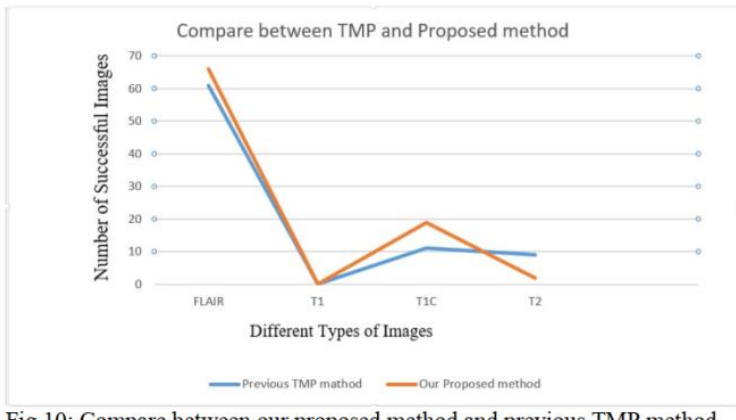

Fig 10: Compare between our proposed method and previous TMP method 


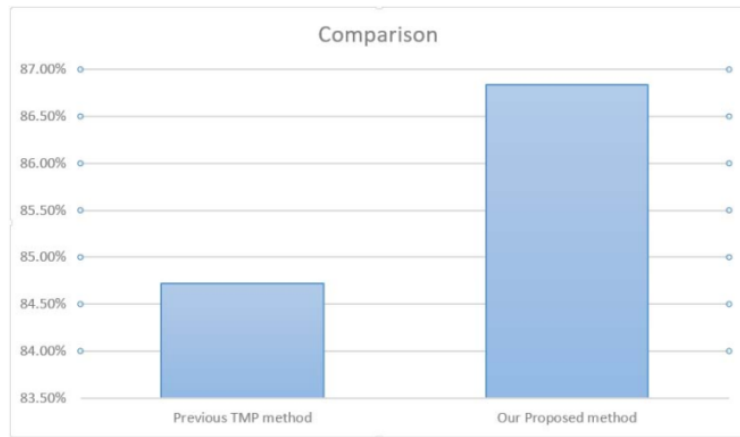

Fig 11: Accuracy comparison between Previous TMP method and Our Proposed method

TABLE 1: PERFORMANCE AND COMPARISON ANALYSIS USING BRATS BRAIN TUMOR DATABASE [7]

\begin{tabular}{|c|c|c|c|}
\hline Algorithm & MRI modalities & Approach & $\begin{array}{c}\text { Accuracy } \\
\text { rate }\end{array}$ \\
\hline Corso 2008 & T1,T1C,T2,FLAIR & Weighted aggregation & $62-69 \%$ \\
\hline Verma 2008 & $\begin{array}{c}\text { T1,T1C,T2,FLAIR } \\
\text {,DTI }\end{array}$ & SVM & $34-93 \%$ \\
\hline Cobzas 2009 & T1C,FLAIR & Level set w/CRF & $50-75 \%$ \\
\hline Menze 2010 & T1,T1C,T2,FLAIR & $\begin{array}{c}\text { Generative model } \\
\text { w/Lesion class }\end{array}$ & $40-70 \%$ \\
\hline Sujan-2016 & FLAIR & $\begin{array}{c}\text { Thresholding and } \\
\text { morphological } \\
\text { Processing }\end{array}$ & $84.72 \%$ \\
& & &
\end{tabular}

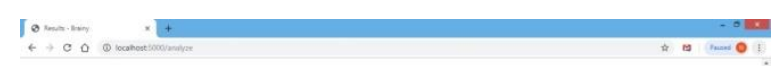

Analysis.
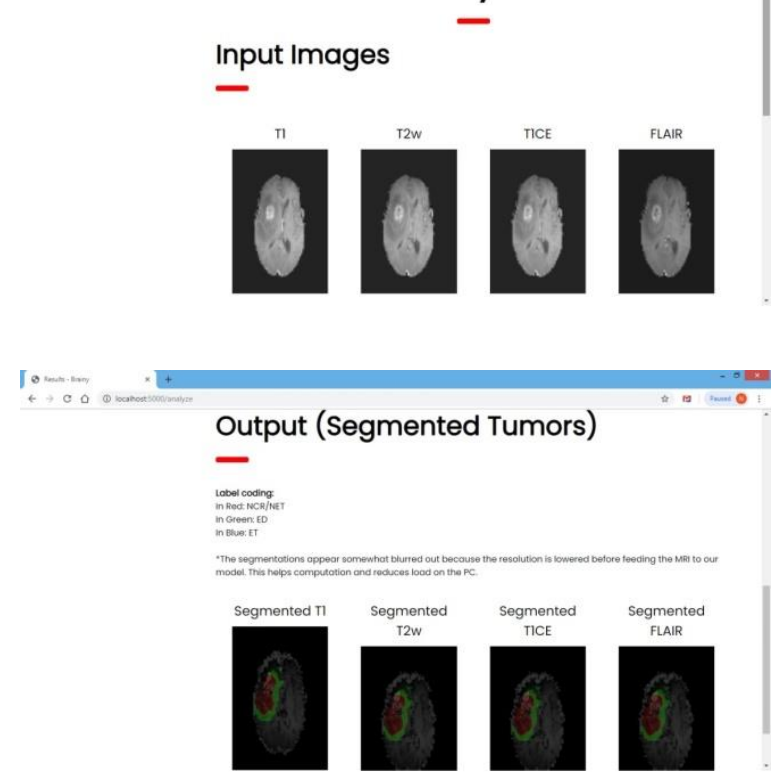


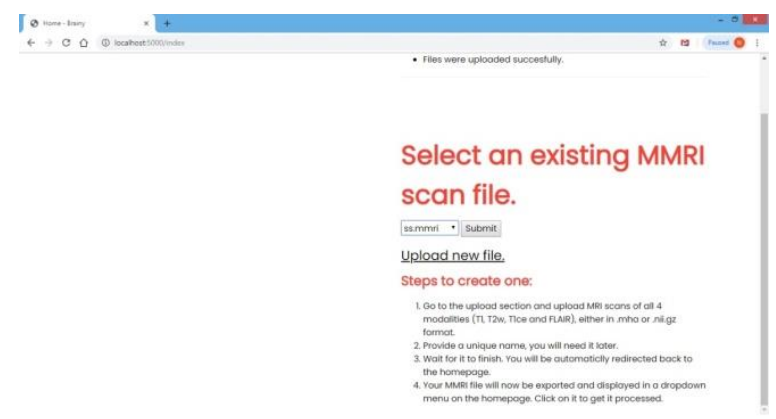

\section{CONCLUSION AND FUTURE SCOPE}

The report concludes that the proposed method is the best way to detect brain tumors. Besides, the level of accuracy has improved compared to previous studies. In our research, the performance of this method is ideal for low noise and very clear images. When it comes to volume reduction and image enhancement, we expect improved accuracy. Besides, it will be important if you can develop effective ways to recognize bad thoughts.

If you could calculate the exact size of a tumor from a 3D image, it would be a lot. Because it helps doctors plan cancer surgery more efficiently and effectively.

\section{REFERENCES}

Tirpude NN*, Welekar RR,“ Effect Of Global Thresholding On Tumor-Bearing Brain MRI Images”, International Journal Of Engineering And Computer Science ISSN:2319-7242 Volume 2 Issue 3 March 2013 Page No. 728-731.

Rohan Kandwal, Ashok Kumar, "An Automated System for Brain Tumor Detection and Segmentation", International Journal of Advanced Research in Computer Science and Software Engineering, Volume 4, Issue 3, March 2014 ISSN: 2277 128X

Jasdeep Kaur, Mamta Garg, "An Improved Weighted Median Filter for the Image Processing Application", International Journal of Science and Research (IJSR), India Online ISSN: 2319-7064

Mr. Rohit S. Kabade, Dr. M. S. Gaikwad, " Segmentation of Brain Tumour and Its Area Calculation in Brain MR Images using Kean

Clustering and Fuzzy C-Mean Algorithm", International Journal of Computer Science \& Engineering Technology (IJCSET). 